\title{
$\angle S$ Research Square \\ Pro-NGF Disrupts Adult Neurogenesis in Humans and Mice Affected by Alzheimer's Disease
}

Bolanle Olabiyi

University of Bonn: Rheinische Friedrich-Wilhelms-Universitat Bonn

\section{Catherine Fleitas}

UdL: Universitat de Lleida

\section{Bahira Zammou}

University of Lleida: Universitat de Lleida

\section{Isidro Ferrer}

University of Barcelona: Universitat de Barcelona

\section{Claire Rampon}

University of Toulouse 3: Universite Toulouse III Paul Sabatier

Joaquim Egea

University of Lleida: Universitat de Lleida

Carme EspinetMestre ( $\square$ carme.espinet@udl.cat )

University of Lleida: Universitat de Lleida https://orcid.org/0000-0001-7172-3407

\section{Research}

Keywords: Alzheimer's disease, adult neurogenesis, pro-NGF, p75, dentate gyrus, memory impairment.

Posted Date: June 29th, 2021

DOI: https://doi.org/10.21203/rs.3.rs-605715/v1

License: (c) (i) This work is licensed under a Creative Commons Attribution 4.0 International License.

Read Full License 


\section{Abstract}

In recent decades, neurogenesis in adult brain has been well demonstrated in a number of animal species, including humans. Interestingly, work with rodents has shown that adult neurogenesis in the dentate gyrus (DG) of the hippocampus is vital for some cognitive aspects, as increasing neurogenesis improves memory while its disruption triggers the opposite effect. Adult neurogenesis declines with age and has been suggested to play a role in impaired progressive learning and memory loss seen in Alzheimer's disease (AD). Therefore, therapeutic strategies designed to boost adult hippocampal neurogenesis may be beneficial for the treatment of $A D$. The precursor forms of neurotrophins, such as pro-NGF, display remarkable increase during $A D$ in the hippocampus and entorhinal cortex. In contrast to mature NGF, pro-NGF exerts adverse functions in survival, proliferation and differentiation. Hence, we hypothesized that pro-NGF and its receptor p75NTR contribute to disrupting adult hippocampal neurogenesis during $A D$. In this study, we took advantage of the availability of mouse models of $A D$ (APP/PS1) and AD human samples to address the role of pro-NGF/p75NTR signalling in different aspects of adult neurogenesis.

Neuroprogenitors of adult mice and human DG samples were identified by immunofluorescence with doublecortin (DCX) antibodies. Interestingly, DCX + progenitors in healthy humans and control animals express p75 neurotrophin receptor (p75NTR). However, this expression is notably decreased in AD conditions. In APP/PS1 mice, memory and cognition were severely impaired. In order to assess the contribution of the pro-NGF/p75NTR pathway to these memory deficits, we injected pro-NGF neutralizing antibodies (ANTI-PRONGF) into the DG of control and APP/PS1 mice which memory was evaluated in Morris water maze test. We observed that anti-pro-NGF injection significantly improved the performance of APP/PS1 animals, but not controls. Interestingly, improved memory in APP/PS1 animals after injection of ANTI-PRONGF correlated with an increase in DCX + progenitors in the DG region of these animals. In summary, our results suggest that pro-NGF is involved in disrupting spatial memory in AD, at least in part by blocking adult neurogenesis. Moreover, we propose that adult neurogenesis alteration could serve as alternative approach towards understanding AD pathology, and additionally offer pro-NGF/p75NTR signalling as a promising therapeutic target.

\section{Background}

The neurodegenerative process involved in Alzheimer's Disease (AD) pathogenesis often begins with memory loss and results in defects in multiple cortical activities later on. It is no longer new that adult neurogenesis occurs in the human brain and contributes to the cognitive functioning of the hippocampus $[1,2]$. Empirical evidence has shown that adult neurogenesis declines with age and that alteration of the neurogenic process contributes to the cognitive deterioration and learning difficulties/memory loss in AD $[3,4,5,6]$.

In the mouse, adult-generated neurons are formed in two main brain regions: the subgranular zone (SGZ) located in the dentate gyrus of the hippocampus, and the subventricular zone (SVZ) of the lateral 
ventricles, which contains lateral, medial, and dorsal microdomains [7]. In the hippocampus, new functional NSCs arise from the adult neural stem cells via the amplification of intermediate progenitors and neuroblasts, followed by the integration of these new neurons into existing neural circuits [8].

Hippocampal neurogenesis has received considerable attention over the recent decades because of its role in learning and memory $[9,10]$. Also, because during $A D$ it has been shown that hippocampal adult neurogenesis is greatly impaired and significantly contributes to learning and memory deficits associated with this pathology $[2,3,4,11]$.

During adult neurogenesis, newly born cells express distinct neurogenic markers characteristic of the different stages of the process. The Primary Neural Progenitor cells (type 1 cells) are located in the subgranular cell layer (SGL) of the DG, where they slowly divide and express stem cell markers such as nestin and Sox2 [18]. They then give rise to intermediate Neuronal Progenitor Cells (type 2 cells), which are more proliferative and have only short processes. Type 2 cells are categorized into type $2 a$ and $2 b$ cells; type $2 \mathrm{a}$ cells express stem cell markers and type $2 \mathrm{~b}$ cells express immature neuronal markers such as doublecortin (DCX), and they in turn give rise to neurons, expressing first calretinin + and then calbindin protein, reflecting their complete differentiation into granular neurons. During the second week after cell division, new-born neurons begin to extend their axonal processes to the CA3 region of the hippocampus and their dendritic processes towards the molecular layer. At this stage, electrophysiological recordings show that they receive direct, slow GABAergic synaptic input and start to show spines on dendrites. Between 4 and 6 weeks after birth, these neurons become fully mature and integrated into the circuit [12, $13,14]$. The decline of adult hippocampal neurogenesis observed with aging and to a greater extent in age-related pathological conditions such as $A D$ suggests that neurogenesis plays a crucial role in proper brain functioning, including learning and memory. Thus, practical approaches to boost adult neurogenesis during neurodegeneration may be a promising strategy to combat the progressive loss of neurons and related cognitive dysfunction associated with $A D$. For this, it is essential to know the exact molecular mechanisms involved in the loss of adult neurogeneisis during AD.

Neurotrophins are a broad family of secreted growth factors that are important for the survival, growth, and maintenance of neurons, with a crucial role in synaptic plasticity, learning and memory $[15,16]$. Of particular interest are nerve growth factor (NGF) and brain derived neurotrophic factor (BDNF). Most neurotrophic factors are synthesized as precursor forms (pro-forms, such as pro-NGF or pro-BDNF) and undergo proteolytic cleavage to render the mature neurotrophic forms Under physiological conditions, the mature form of neurotrophins is generally produced from the intracellular activities of proteases such as convertases to elicit their trophic functions. Numerous studies have shown that proneurotrophins may be secreted without being cleaved, and this has deleterious effects on neuronal function $[5,6,17,18,19,20]$. The pro and mature forms of these neurotrophins have different affinities and specificities for various receptors(such as the p75 neurotrophic receptor (p75NTR). p75NTR belongs to the TNF family (tumour necrosis factor) of death receptors but has no catalytic activity. It has multiple roles in cellular homeostasis, apoptosis, cell cycle arrest, survival and proliferation. Depending on the ligand and coreceptor, p75NTR can perform any of the functions mentioned above [21]. When bound by mature 
neurotrophin, it interacts with Trk receptors to promote cell survival. Still, when bound by precursor forms such as proNGF and proBDNF, it interacts with sortilin to form p75NTR-sortilin receptor complex, and death signalling is activated [22]. Among several elements involved in propagating this death signal, the pathway includes the induction of NRAGE and NRIF, which facilitates apoptosis via the JNK kinases [21, $22,23]$. Another critical route of this death signalling involves the processing of $p 75$ Intracellular domain by two secretases, $a$ and $\gamma$. When proNGF or proBDNF binds to the p75NTR/sortilin complex, the $a-$ secretase begins the death process by cleaving the extracellular domain of $p 75$, leaving the rest of the receptor bound to the membrane. The $y$-secretase then cleaves the cytoplasmic domain of the receptor, thereby releasing the $20 \mathrm{kDa}$ intracellular domain fragment (p75 ICD). The p75ICD, through its interaction with TRAF6 and NRIF, subsequently translocate to the nucleus and facilitates the expression of proapoptotic genes $[18,24,25,26,27]$.

Interestingly, several studies have implicated the precursor forms of the neurotrophins in many aspects of the pathogenesis of $A D$, especially in cell death $[17,18,19,24]$. For instance, in a previous study, we described how ICV injection of proNGF elicited spatial memory deficits in control mice, similar to those observed in AD mouse models such as the APP/PS1 mice [18]. This effect is probably exacerbated by the observation that under pathological conditions involving oxidative stress, such as in AD, proneurotrophins may undergo AGE-derived modifications that promote their stability and facilitate the death signalling cascade $[18,19,24]$. However, it is not clear whether the pro-neurotrophin/p75NTR signalling is involved in other processes related to the AD pathology. In the present study, we investigated the contribution of the pro-NGF/p75NTR signalling pathway in adult hippocampal neurogenesis in AD patients and in APP/PS1 transgenic mouse model for $A D$ and its impact in the cognitive dysfunctions of the disease.

\section{Methods}

\section{Human brain samples}

Brain samples were obtained from the Institute of Neuropathology, Bellvitge University Hospital. Brain tissues were obtained from the Institute of Neuropathology HUB-ICO-IDIBELL Biobank and the Hospital Clinic-IDIBAPS Biobank following the guidelines of Spanish legislation on this matter (Real Decreto de Biobancos 1716/2011) and approval of the local ethics committees. For autopsy, one hemisphere was rapidly cut in $1 \mathrm{~cm}$ thick coronal sections, and selected areas of the encephalon were dissected, frozen on dry ice, and stored at $-80^{\circ} \mathrm{C}$ in labelled plastic bags until use. The other hemisphere was fixed by immersion in $4 \%$ buffered formalin for three weeks for morphologic examination. The neuropathological study was carried out on twenty-five regions of the cerebral cortex, diencephalon, thalamus, brainstem, and cerebellum. De-waxed paraffin sections were stained with haematoxylin and eosin and KlüverBarrera, then processed for immunohistochemistry of microglia-specific markers, glial fibrillary acidic protein, $\beta$-amyloid, phosphorylated tau, a-synuclein, TDP-43, ubiquitin, and p62. Neuropathological diagnosis of AD was carried out following the Braak and Braak stages [28] adapted to paraffin sections 29]. Cases with concomitant pathologies, including Lewy body diseases, tauopathies (particularly argyrophilic grain disease), vascular diseases, TDP-43pathies, and metabolic syndrome were excluded. 
Control and disease cases were processed in parallel. The anterior hippocampus area was used for further immunohistochemical studies.

\section{Animal model}

Considering that amyloid precursor protein (APP) and Presenilin 1 (PS1) are mutated in $78 \%$ and $18 \%$ of the familial cases of Alzheimer, respectively, the double transgenic APP/PS1 mouse model has been one of the most commonly used models in the study of Alzheimer's disease [30, 31, 32].

APPswe/PS1dE9 with a C57BL/6 background (double transgenic mice expressing a chimeric mouse/human amyloid precursor protein and a mutant human PS1 with deletion in exon 9) were purchased from the Jackson Laboratory and kept in a specific pathogen-free environment under standard animal housing conditions in a $12 \mathrm{hrs}$ dark-light cycle with free access to food and water in the animal house facility of the Universitat de Lleida. Heterozygous males were bred with wild-type C57BL/6 females. Animal procedures were conducted according to ethical guidelines (European Communities Council Directive 86/609/EEC) and approved by the local ethics committee of the Universitat de Lleida. For experiments, tail biopsies were taken from P0 offspring for genotyping by PCR according to the PCR conditions suggested by the Jackson Laboratory. Mice not expressing the transgene were used as controls.

\section{Behavioural test: Morris water maze}

The experimental animals were subjected to Morris water maze (MWM) test to assess their cognition/memory associated with hippocampal function [33,34]. All of the behavioural procedures were conducted at the same time of day, in an isolated room every day during 4 days. The mice were trained to find a $50 \mathrm{~cm}^{2}$ platform hidden under the water surface in a water tank of $150 \mathrm{~cm}$ diameter, with four different geometrical forms attached to the four sides of the water tank wall. Four trials per day with start positions close to the four geometrical signs were carried out, and latency in reaching the platform was recorded. Cut-off time to find the platform was 90 seconds, and mice failing to find the platform were placed on it and left there for 15 seconds. Each trial for a single animal was 30 minutes apart from the previous [18].

\section{Stereotaxic injection}

8-9 months old female and male APPsw695/PS1 dE9 mice and control animals were placed under isoflurane anaesthesia for a couple of minutes, after which they were injected intraperitoneally with the analgesia Rompun (2\%) and ketamine $(10 \%)$ in $0.9 \% \mathrm{NaCl}$. Then, mice were fixed securely unto the stereotaxic apparatus, and the skull was carefully exposed. The stereotaxic apparatus was then adjusted to the accurate position after which a metal bar driller was slowly used to make a hole in the skull. $5 \mu \mathrm{l}$ per DG of the anti proNGF antibody or BSA control solution was gradually injected bilaterally into the dentate gyrus according to the stereotaxic coordinates (Bregma: anteroposterior: $2 \mathrm{~mm}$, lateral: $+/-1.6$ $\mathrm{mm}$, dorsolateral: $2.5 \mathrm{~mm}$ from the skull), at a speed of $1 \mu \mathrm{l} / \mathrm{min}$. After the successful injection, the needle 
was held in place for another minute before it was retracted slowly at the same rate of $1 \mu \mathrm{l} / \mathrm{min}$ speed. After this, the incisions were stitched, and the mice were returned to their home cages for a recovery period of 10 days.

Another set of APP/PS1 transgenic mice were stereotaxically injected with enhanced green fluorescent protein (eGFP) expressing the Moloney leukaemia-derived retroviral vector pCMMP-IRES2eGFP-WPRE. This was carried out using the previously described protocol [3].

\section{Immunohistochemistry}

A week after the behavioural study, the mice were anesthetized with Rompun (2\%, Bayer)/ (Ketamine $0.1 \mathrm{mg} / \mathrm{g}$, Merial) and transcardially perfused with $\mathrm{NaCl}(9 \%)$ followed by $4 \%$ PFA in PBS. Brains were dissected and fixed overnight in 4\% PFA in PBS, then were washed with PBS 3 times for 10 minutes and subsequently cryoprotected in 30\% sucrose (Scharlau) in PBSO/N, embedded in cryoprotective tissuefreezing medium (General Data), and stored at $-80^{\circ} \mathrm{C}$. $30 \mu \mathrm{M}$ serial coronal sections (cryosections) were then made using a cryostat (Leica CM 3000), and collected in Super frost PlusTM slides (ThermoFisher) and stored at $-80^{\circ} \mathrm{C}$ for further use.

The immunohistochemistry protocol followed in this study varied slightly according to the sets of samples analysed: hippocampal slices obtained from blocking a-proNGF stereotaxically injected mice, GFP retroviral-injected mice, or human samples. The procedures are summarized as follows:

\section{Immunostaining protocol for human samples}

Human samples were deparaffined by heating them to $62^{\circ} \mathrm{C}$ for $30 \mathrm{~min}$ and then substituted in xylol 2 times at 10-minute intervals. A series of ethanol washes were then done in the lamina flow hood at varying concentrations as follows: 2 times in ethanol 100\%, 2 times in ethanol 95\%, and 2 times in ethanol $60 \%$; the interval between each of these washes was 5 minutes. The resulting samples were rinsed in distilled water for 10 min, followed by 3 10-minute washes in PBS 1X and then 1 10-minute wash in 50mM NH4Cl+PBS1X. Lipofusion was done under black conditions to eliminate autofluorescence by blocking in black Sudan suspension for 25 minutes at room temperature before antigen retrieval (10-minutes wash in preheated Tris- $\mathrm{HCl}$ buffer $20 \mathrm{mM} \mathrm{pH} 9.5$ at $97^{\circ} \mathrm{C}$ for 20 minutes), after which it was cooled down. The next step was to wash it in $0.1 \%$ PBST 2 times for 15 minutes and block with $5 \%$ donkey serum for 1 hour. Primary antibodies were then added at working concentration 1:100 and kept at $4^{\circ} \mathrm{C}$ till the next day (rabbit DCX Santa Cruz; goat anti-p75ECD R and D). On the following day, having spent 28 hours in the cold room, the samples were left at room temperature for 2 hours before proceeding with the 3 washes in PBST $0.1 \%$ at 15 -minute intervals. The samples were then incubated with secondary antibodies (working dilution 1: 500 in blocking solution) for 2 hours. They were then washed 3 times in PBS Tween $0.1 \%$ and a last washing in $0.1 \%$ PBS before they were finally mounted with the coverslip using the mounting medium Fluoromount G (Southern Biotech 0100-01).

\section{Immunostaining procedure for mouse cryosections.}


The sections were consequently subjected to one-hour blocking and permeabilization process with PBST and $1 \%$ donkey serum (5\%) (Jackson 017-000-121 IR), then incubated with primary antibodies (in blocking solution 1:100) (rabbit DCX Santa Cruz; goat anti-p75ECD R and D), and subsequently kept at $4^{\circ}$ $C$ for 5 days. On the 5 th day, the samples were removed from the cold room and kept at room temperature for 2 hours. After that, they were washed three times with PBST $0.1 \%$ at 10 -minute intervals; and then incubated with secondary antibodies in blocking solution (Working dilution 1:500 plus DAPI for 2 hours at room temperature). Finally, the cells were washed three more times with PBST $0.1 \%$ for 15 minutes, after which they were mounted with coverslips using Fluoromount G (Southern Biotech 0100-01).

\section{Immunostaining protocol for GFP retroviral injected hippocampal slices}

The mouse hippocampal slices were subjected to immunohistochemistry protocol performed in batches in round-bottomed Eppendorf tubes. The slices were first placed at room temperature for 40 minutes, followed by 4 free-floating washes with PBST ( $0.25 \%$ Triton) of 5 minutes each. After that, they were washed 3 times in PBST (0.1\%) at 10 min intervals and then in PBST0.1\%+NH4Cl $50 \mathrm{mM}$ for 10 minutes. Antigen retrieval was done using pre-heated citrate buffer and the antigens were then placed in $95^{\circ} \mathrm{C}$ boiling water for $30 \mathrm{~min}$. The slices were cooled for about 15 minutes and washed 2 times in PBST $(0.1 \%$ Triton) to remove remains of the fixing solution. Then, they were incubated with the blocking solution following the protocol described in the section below but in batches in the Eppendorf tubes. The antibodies were used in blocking solution at 1:100 (goat anti-p75ECD R and D; rabbit anti-DCX Santa Cruz). After that, they were washed three times with PBST $0.1 \%$ at 10 min intervals, and then incubated with secondary antibodies in blocking solution (working dilution 1:500 plus DAPI for 2 hours at room temperature). Finally, the slices were carefully transferred from Eppendorf tubes to super frost glass slides and mounted with coverslips using Fluoromount G (Southern Biotech 0100-01). Fluorescence images were acquired on a confocal microscopy setup (Olympus FV1000, 60X PlanApo using software Fluoview v.4.3) or on an inverted fluorescence microscope (OlimpusIX71, 20X LCPlanFI).

All microscopes were set to collect images below saturation and were kept constant for all images taken in one experiment.

\section{Statistical analysis}

Statistical analysis of data was performed using SPSS statistical software (SPSS for Windows, v.16, SPSS, Inc., Chicago, IL). Student's t-test and ANOVA were used to compare quantitative with qualitative variables and $\chi 2$ test was used for categorical variables. Values were expressed as mean $\pm S D$, and statistical significance are indicated as follows; $p<0.05(*), p<0.01$ (**) or $p<0.001(\star \star \star)$.

\section{Results}

\section{Human and mouse hippocampal NSCs express p75NTR}


The existence of adult neurogenesis in human DG samples and its decrease in AD have recently been evidenced [1]. The mechanisms regulating such a decrease in AD remain widely unknown. In previous studies, we and others reported a significant increase in pro-neurotrophins in the hippocampus of $A D$ patients $[5,6,17,18]$. However, as we initially reported in the hileal region, the expression levels of p75NTR in granular cells was high and not affected in $A D$ ([9] and data not shown). Similarly, the coreceptor of p75NTR, sortilin, involved in signalling apoptosis was ubiquitously expressed in human DG, and no changes in the expression pattern were detected in AD (Supplementary data, Fig. 1).

To determine whether the apoptotic effect of pro-neurotrophins through their interaction with p75NTR, could account for the decrease of NSCs in human DG of AD subjects, we quantified the expression of DCX, a specific marker for immature neurons- and its co-localization with p75NTR (Fig. 1) in the DG. Our results show that DCX/p75NTR are co-localized in the control DG human samples to a substantial extent (Fig. 1A, B). In AD samples, however, DCX/p75NTR co-localization was significantly reduced (Fig. 1A, B). To further characterize the possible role of p75NTR as signalling apoptotic death in newly generated neurons in the AD context, we used the AD mouse model APP/PS1. We used retroviral GFP delivery to label NSCs in control and APP/PS1 mice and after 21 dpi (days post-injection), immunohistochemistry against GFP and p75NTR were carried out to determine the extent of GFP+/p75NTR + co-localization. We observed that most of the GFP + cells expressed p75NTR in the DGs of control mice (Fig. 2A, B). However, similar to happen in human AD samples, this co-localization was strongly reduced in APP/PS1 mice. Considering our previous results where we did not find significant differences in p75NTR expression in AD models (9); these results in human and mouse $A D$ samples are interesting and suggest a relationship between cell survival and p75NTR expression specifically in neuroprogenitor cells.

\section{Injection of proNGF blocking antibody enhances memory function in transgenic APP/PS1 mice}

Behavioural and memory alterations are well described in APP/PS1 mice along disease progression [30]. Interestingly, we demonstrated that some of these cognitive defects, including an increase of apoptosis and defects in spatial memory (by MWM), could be recapitulated in control mice by ICV injection of proNGF, either purified from human AD-affected hippocampus or recombinant and AGE-modified in vitro [18]. We therefore hypothesized that pro-NGF might have an important role during the development of the disease in the APP/PS1 AD mice and wanted to examine whether ablation of pro-NGF in the DG of APP/PS1 mice could abolish their spatial memory deficits. Thus, we injected these mice with either antipro-NGF neutralizing antibodies $(5 \mu \mathrm{g} / \mathrm{DG})$ or BSA control solution $(5 \mu \mathrm{g} / \mathrm{DG})$ bilaterally in the $\mathrm{DG}$, after which we allowed the mice to fully recover from the injection before subjecting them to the same behavioural tasks as before the injection. The amount of anti-proNGF antibody that we injected in the present study ( $5 \mu \mathrm{g} / \mathrm{DG}$ ) should be enough to block the increase in proNGF found in the AD hippocampus. This experiment was intended to determine the effect of blocking proNGF activity on adult hippocampal neurogenesis and memory (by Morris Water Maze. APP/PS1 group of mice injected with control BSA-saline solution, clearly display a memory delay (difference between day 1 and 4 is no significative, ANOVA $p=0,15$ ) compared to the control group which rapidly decrease the latency over the days of training $(p=0,0003)$ (Fig. 3A). Noticeably, injection of anti-pro-NGF antibodies in APP/PS1 mice 
strongly reduced time latency and the transgenic mice performed almost as good as the control group over the time of training (Fig. 3B) (difference between day 1 and 4 is no significative, ANOVA $p=0,15$ ). For the control group, no significant differences were observed between injection with anti-pro-NGF or BSA ( $p$ $=0,0001$ (Fig. 3B, 3C). Taken together, our results suggest that memory loss of APP/PS1 mice is at least mediated by pro-NGF as injection of anti-pro-NGF, significantly improved the memory tasks of these animals.

\section{Injection of proNGF blocking antibody enhances neurogenesis in transgenic APP/PS1 mice}

We next evaluated if anti-proNGF injection in APP/PS1 mice could have an effect on adult neurogenesis. For this, we perfused the mice after injection, at the end of the Morris Water Maze training period, and using DAPI, anti-p75NTR, and anti-DCX antibodies we assessed co-localization patterns. APP/PS1 mice showed lower expression of DCX and lower co-localization of P75NTR/DCX compared to controls when injected with BSA (Fig. 4). In contrast, anti-proNGF injection in APP/PS1 mice, rescued significantly the percentage of DCX + cells, although the percentage of DCX/p75NTR was the same as in BSA-injected animals (Fig. 5A, B, D). These experiments demonstrate that inhibition of pro-NGF brings back the percentage of DCX + cells to control levels, thus bursting neurogenesis in these APP/PS1 animals. This suggests that decreased proNGF in DG in APP/PS1 animals may allow the development of NSCs, mainly those expressing less p75 (Figs. 5B and 5D). At this point, it is tempting to speculate that this improvement of adult neurogenesis is in part responsible for the gain of spatial memory in these anti-proNGF-treated APP/PS1 mice (see discussion below).

\section{Discussion}

It has been noted over the years that high concentration of pro-neurotrophins significantly contribute to the pathogenesis of Alzheimer's disease [5, 6, 17, 18, 19, 35]. Among other factors, oxidative stress, inflammation, excitotoxicity, and altered adult hippocampal neurogenesis have been suggested to play a role in the progression of $\operatorname{AD}[1,5,24,36,37]$. In this study, we investigated the impact of variations in proNGF signalling on adult hippocampal neurogenesis in a mouse model of $A D$ as well as in human brain samples from AD patients.

It is now well known that neurogenesis occurs in the DG of adult humans and that the process persists but is affected in AD patients. During the multistep process of neurogenesis, DCX resides at the crossroads of new neuron formation. It has been demonstrated that the expression of this marker sends a neurogenic signal to immature neural progenitor cells and contribute to instruct them to fully differentiate into mature neurons. As such, DCX has been widely used for the study of neurogenesis [1, 10]. The colocalization of DCX and p75 examined in this study is of great importance because p75 is expressed in the hippocampus and also participates in the death signalling pathway of proneurotrophins. Thus, its ambivalent role motivates the need to understand its co-expression pattern with DCX (a typical marker of neurogenesis). 
Besides and as previously reported by our group $[6,17,18,19]$, the ligands of the $p 75 /$ sortilin signalling pathway of apoptosis, proNGF and proBDNF, are in general, present in greater quantities in AD human brain and more significantly, in the DG and hilar region of the hippocampus. Therefore, studying the expression of their receptors in the NSCs of the DG is also relevant to the examination of neurogenesis.

Our findings reveal that p75 colocalization with DCX is sharply decreased in the DG of AD patients compared to healthy controls. We hypothesized that the decrease in DCX/p75 and DCX/sortilin colocalization in $A D$ patients could be due to the death of the neurons initially expressing $D C X$. In other words, this might also mean that the marked decline in adult neurogenesis observed in AD patients could be related to neuronal degeneration/death mediated by the proneurotrophin/p75 signalling pathway.

After several contradictory reports regarding whether or not adult neurogenesis occurs in humans, Moreno-Jiménez and colleagues nicely demonstrate that it does occur in human [1]. The present work supports their findings in providing further evidence of its occurrence in humans. Mention here these evidences and connect to the next paragraph.

Consistent with the reduction in the number of neurons co-expressing $D C X / p 75$ that we observe in $A D$ patients, we report the same trend in the APP/PS1 mouse model of AD. The choice of our animal model was prompted by the fact that in familial $A D$, more than half of the cases have inherited mutations in the presenilin 1 secretase and amyloid precursor protein (APP) [38]. The deletion in exon 9 of PS1 coupled with defective APP has been identified as the significant genetic error associated with this form of AD.

Based on this, we studied the neural stem cells of the DG of these APP/PS1 mice. Our immunohistochemical work indicates that neural stem cells of APP/PS1mice show less co-expression of p75 and DCX, just as is the case in AD patient's samples. The evidence of adult neurogenesis in mice is less controversial; numerous studies have already established its existence in mice $[3,4,8]$. Using a GFPexpressing retroviral vector, we screened for $p 75$ expression in dividing neural stem cells of APP/PS1 mice. We found that less GFP-labeled cells expressed p75 in AD mice compared to their wild-type counterparts. Our inference from these three experiments boils down to neurogenesis as a significant process that is impaired in AD.

Here we provide evidence that neurogenesis plays a crucial role in maintaining the granular neurons population in the DG of healthy subjects. When this process is altered by neurodegeneration, it could lead to an acceleration of neuronal death via the action of the proneurotrophin/p75 pathway.

Neurodegenerative diseases share common features including neuronal death and behavioural abnormalities. In Alzheimer's disease, memory and cognitive deterioration pose a serious threat to the wellbeing of the aging population. We showed that AD mice exhibited lower cognitive function based on the results obtained from the Morris water maze test which have been used in previous studies to monitor the cognitive behaviour of animals and also to assess their learning abilities [19, 33, 34, 39]. Using the same mice model, other behavioural tests have been used $[3,4]$ to confirm that the loose of cognitive 
function in the disease is linked to the reduction of the number and the maturation degree of NSCs in the DG.

The implications of the roles of proneurotrophins and the p75 signalling pathway in the pathogenesis of $A D$ that emerged from previous findings in our lab and others finally prompted us to examine the possible effects of inhibiting proNGF activity on the cognitive function of AD mice. In the present study we found that after injecting proNGF-blocking antibody into the DG of the APP/PS1 mice, their memory and proportion of NSCs were greatly enhanced. We suggest that proNGF is partly involved in causing the cognitive deficits in the APP/PS1 mice and in disruption of human adult neurogenesis.

In conclusion, NSCs of the dentate gyrus of control human samples express DCX and this expression significantly decreases in AD patients. The NSCs express p75NTR which colocalizes with DCX to a greater extent in the control than in the AD brain. Neurogenesis is diminished in the NSCs of AD patients and APP/PS1 mice model. APP/PS1 mice experience behavioural abnormalities associated with cognitive deficits. Upon administration of proNGF-blocking antibodies, the number of NSCs and the cognitive function of the AD mice were both greatly enhanced. The proneurotrophin/p75 signalling pathway has a negative impact on cognitive function and adult hippocampal neurogenesis. Hence, our findings, further support the idea that adult neurogenesis could serve as an alternative therapeutic target in the pathogenesis of $A D$.

\section{Abbreviations}

$A D$

Alzheimer's disease; BSA:Bovine serum albumin; ICV:intra cerebro-ventricular; DCX:doublecortin; ND; neurodegeneration; NSCs:neural stem cells; P/S:penicillin/streptomycin; PBS:phosphate buffered saline; PBS-T:PBS with 0, 1\% Triton X-100; PFA:paraformaldehyde; proNGF:precursor form of NGF; PS1:presenilin 1; p75NTR:p75 neurotrophin receptor; SD:standard deviation; TBS-T:50 mM Tris, pH 8.0; $133 \mathrm{mM} \mathrm{NaCl}, 0.2 \%$ Tween 20.

\section{Declarations}

The authors declare that they have no competing interests.

\section{Ethics approval and consent to participate}

Brain samples were obtained from the Institute of Neuropathology, Bellvitge University Hospital. Brain tissue was obtained from the Institute of Neuropathology HUB-ICO-IDIBELL Biobank and the Hospital Clinic-IDIBAPS Biobank following the guidelines of Spanish legislation on this matter (Real Decreto de Biobancos 1716/2011) and approval of the local ethics committee.

Animal procedures were conducted according to ethical guidelines (European Communities Council Directive 86/609/EEC) and approved by the local ethics committee of the Universitat de Lleida. 


\section{Consent for publication}

Not applicable.

\section{Availability of data and materials}

Data sharing is not applicable to this article as no datasets were generated or analyzed during the current study.

\section{Competing interests}

The authors declare that they have no competing interests.

\section{Funding}

This work was supported by “Fundació La Marató 2015" (C.E.). We thank, IRBLleida Biobank (B.0000682), PLATAFORMA BIOBANCOS PT13/0010/0014, HUB-ICO-IDIBELL Biobank for providing human tissue, and UAI IRBLleida for management support.

\section{Authors' contributions}

All authors have participated in the study and take public responsibility for appropriate portions of the content, and have agreed to be accountable for all aspects of the work in ensuring that questions related to the accuracy or integrity of any part of the work are appropriately investigated and resolved. FC and OB are responsible for the most of the design of the study and of data acquisition, analysis, and interpretation. ZB contributed to the experimental work. $\mathrm{Fl}$ is responsible for the obtaining and analysis of the human brain samples. RC is responsible for the obtaining of the GFP retrovirally-injected mouse samples. RC, EJ, and EC were involved in the drafting of the manuscript and its critical review. All authors read and approved the final manuscript.

\section{Aknowledgments}

We thank Ester Aso for caring for transgenic mice, Jesus Moreno for helping in the management of human post-mortem samples, and Eila Vicedo and Nuria Terés for helping with various aspects of the experimental work. We thank Sonia Rius and the animal house facility staff of the University of Lleida for technical assistance.

\section{References}

1. Moreno-Jiménez EP, Terreros-Roncal J, Flor-García M, Rábano A. Llorens-Martín M.J Evidences for adult hippocampal neurogenesis in humans. Neurosci. 2021;Mar 24(12):2541-53. 41 (.

2. Moreno-Jiménez EP, Flor-García M, Terreros-Roncal J, Rábano A, Cafini F, Pallas-Bazarra N, Ávila J, Llorens-Martín M. Adult hippocampal neurogenesis is abundant in neurologically healthy subjects and drops sharply in patients with Alzheimer's disease. Nat Med. 2019;Apr;25(4):554-60. 
3. Richetin K, Leclerc C, Toni N, Gallopin T, Pech S, Roybon L, Rampon C. Genetic manipulation of adultborn hippocampal neurons rescues memory in a mouse model of Alzheimer's disease. Brain. 2015;138(2):440-55.

4. Richetin K, Petsophonsakul P, Roybon L, Guiard BP, Rampon C. Differential alteration of hippocampal function and plasticity in females and males of the APPXPS1 mouse model of Alzheimer's disease. Neurobiol Aging. 2017;57:220-31.

5. Dard RF, Dahan L, Rampon C. Targeting hippocampal adult neurogenesis using transcription factors to reduce Alzheimer's disease-associated memory impairments. Hippocampus. 2018; 1-22.

6. Fahnestock M, Michalsk B, Xu B, Coughlin MD. The precursor pro-nerve growth factor is the predominant form of nerve growth factor in brain and is increased in Alzheimer's disease. Molecular Cellular Neurosciences. 2001;18(2):210-20.

7. Apple DM, Fonseca RS, Kokovay E. The role of adult neurogenesis in psychiatric and cognitive disorders. Brain Res. 2016;1655:1-9.

8. Toda T, Parylak SL, Linker SB, Gage FH. The role of adult hippocampal neurogenesis in brain health and disease. Mol Psychiatry. 2019;24(1):67-87.

9. Eriksson PS, Perfilieva EK, Eriksson TB, Alborn AN, ORdborg C, Peterson DA, Gage FH. Neurogenesis in the adult human hippocampus. Nat Med. 1998;4(11):1313-7.

10. Knoth R, Singec I, Ditter M, Pantazis G, Capetian P, Meyer RP, Horvat V, Volk B, Kempermann G. Murine features of neurogenesis in the human hippocampus across the lifespan from 0 to 100 years. PLoS ONE. 2010;5(1):e8809; 1-10.

11. Peng $L$, Bonaguidi MA. Function and dysfunction of adult hippocampal neurogenesis in regeneration and disease. Am J Pathol. 2018;188(1):23-8.

12. Deng W, Aimone JB, Gage FH. New neurons and new memories: how does adult hippocampal neurogenesis affect learning and memory? Nat Rev Neurosci. 2010;May;11(5):339-50.

13. Aimone JB, Deng W, Gage FH. Adult neurogenesis: integrating theories and separating functions. Trends Cogn S. 2010; Jul;14(7):325-37.

14. Vivar C, van Praag H. Functional circuits of new neurons in the dentate gyrus. Front Neural Circuits. 2013; Feb 25; 7:1515. Barde YA. Trophic factors and neuronal survival. Neuron 1989; 2(6): 1525-34.

15. Barde YA. Trophic factors and neuronal survival. Neuron. 1989;2(6):1525-34.

16. Chao MV, Rajagopal R, Lee FS. Neurotrophin signalling in health and disease. Clin Sci (Lond). 2006;110(2):167-73.

17. Pedraza CE, Podlesniy P, Vidal N, Arévalo JC, Lee R, Hempstead B, Ferrer I, Iglesias M, Espinet C. ProNGF isolated from the human brain affected by Alzheimer's disease induces neuronal apoptosis mediated by p75NTR. Am J Pathol. 2005;166(2):533-43.

18. Podlesniy P, Kichev A, Pedraza C, Saurat J, Encinas M, Perez B, Ferrer I, Espinet C. Pro-NGF isolated from the human brain affected by Alzheimer's disease induces neuronal apoptosis mediated by p75NTR. Am J Pathol. 2006;166(2):533-43. 
19. Kichev A, Ilieva EV, Piñol-Ripol G, Podlesniy P, Ferrer I, Portero-Otín M, Espinet C. Cell death and learning impairment in mice caused by in vitro modified pro-NGF. Am J Pathol. 2009;175(6):257485.

20. Lee R, Kermani P, Teng KK, Hempstead BL. Regulation of cell survival by secreted proneurotrophins. Science. 2001;294:1945-8.

21. Hempstead BL. The many faces of p75NTR. Curr Opin Neurobiol. 2002;12(3):260-7.

22. Nykjaer A, Lee R, Teng KK, Jansen P. Sortilin is essential for proNGF-induced neuronal cell death. Nature. 2004;427:843-8.

23. Barker PA. p75NTR Is positively promiscuous: novel partners and new insights. Neuron. 2004;42:529-33.

24. Fleitas C, Piñol-Ripoll G, Marfull P, Rocandio D, Ferrer I, Rampon C, Egea J, Espinet C. ProBDNF is modified by advanced glycation end products in Alzheimer's disease and causes neuronal apoptosis by inducing p75 neurotrophin receptor processing. Molecular Brain. 2018;11(1):1-16.

25. Frade JM. Nuclear translocation of the p75 neurotrophin receptor cytoplasmic domain in response to neurotrophin binding. J Neurosci. 2005;25(6):1407-11.

26. Parkhurst CN, Zampieri N, Chao MV. Nuclear localization of the p75 neurotrophin receptor intracellular domain. J Biol Chem. 2010;285(8):5361-8.

27. Kenchappa RS, Zampieri N, Chao MV, Barker PA, Teng HK, Hempstead BL, et al. Ligand-dependent cleavage of the P75 neurotrophin receptor is necessary for NRIF nuclear translocation and apoptosis in sympathetic neurons. Neuron. 2006;50:219-32.

28. Braak H, Alafuzoff I, Arzberger T, Kretzschmar H, Del Tredici K. Staging of Alzheimer diseaseassociated neurofibrillary pathology using paraffin sections and immunocytochemistry. Acta Neuropathol. 2006;112:389-404.

29. Braak H, Braak E. Demonstration of amyloid deposits and neurofibrillary changes in whole brain sections. Brain Pathol. 1991;1:213-6.

30. Sasaguri H, Nilsson P, Hashimoto S, Nagata K, Saito T, De Strooper B, et al. APP mouse models for Alzheimer's disease preclinical studies. EMBO J. 2017; Sep 1;36(17):2473-2487.

31. Borchelt DR, Thinakaran G, Eckman CB, Lee MK, Davenport F, Ratovitsky T, et al. Familial Alzheimer's disease-linked presenilin 1 variants elevate Abeta1-42/1-40 ratio in vitro and in vivo. Neuron. 1996;17:1005-13.

32. Aso E, Lomoio S, López-González I, Joda L, Carmona M, Fernández-Yagüe N, et al. Amyloid generation and dysfunctional immunoproteasome activation with disease progression in animal model of familial Alzheimer's disease. Brain Pathol. 2012;22(5):636-53.

33. Schoenfeld R, Schiffelholz T, Beye C, Leplow B, Foreman N. Variations of the Morris water maze task to comparatively assess human and rodent place navigation. Neurobiol Learn Mem. 2017;139:11727. 
34. Garthe A, Kempermann G. An old test for new neurons: refining the Morris water maze to study the functional relevance of adult hippocampal neurogenesis. Front Neurosci. 2013; May 3; 7: 63.

35. Fahnestock M, Shekari A. ProNGF and neurodegeneration in Alzheimer's disease. Frontiers in Neuroscience. 2019;13(FEB):1-11.

36. Espinet C, Gonzalo H, Fleitas C, Menal MJ, Egea J. Oxidative stress and neurodegenerative diseases: a neurotrophic approach. Curr Drug Targets. 2015;16(1):20-30.

37. Collin F, Cheignon C, Hureau C. Oxidative stress as a biomarker for Alzheimer's disease. Biomark Med. 2018 Mar;12(3):201-3.

38. Lanoiselée HM, Nicolas G, Wallon D, Rovelet-Lecrux A, Lacou M, Rousseau S, Campion D. APP. PSEN1, and PSEN2 mutations in early-onset Alzheimer disease: A genetic screening study of familial and sporadic cases. PLoS Medicine. 2017;14(3):1-16.

39. Gil-Mohapel J, Brocardo PS, Choquette W, Gothard R, Simpson JM, Christie BR. Hippocampal neurogenesis levels predict watermaze search strategies in the aging brain. PLoS One. 2013;Sep 23(9):e75125. 8 ).

\section{Tables}

Table 1

Summary of the patients from whom hippocampal samples were obtained and studied. C: control, AD: Alzheimer's disease;

$A, B$ and $C$ : Braak and Braak's classification of AD stages

depending on amyloid plaques; 0-V: Braak and Braak's classification of $A D$ stages depending on the distribution and number of neurofibrillary tangles.

\begin{tabular}{|llllll|}
\hline N & Age & Gender & Diagnosis & Braak & Post-mortem \\
\hline 1 & 46 & F & C & 0 & 9 \\
\hline 2 & 47 & M & C & 0 & 5 \\
\hline 3 & 24 & F & C & 0 & 6 \\
\hline 4 & 79 & M & AD & V/B & 5 \\
\hline 5 & 82 & F & AD & V/B & 2 \\
\hline 6 & 79 & M & AD & V/C & 7 \\
\hline 7 & 85 & F & AD & VI/C & 12 \\
\hline
\end{tabular}


Table 2

Demographic and clinical characteristics of the patients from whom CSF samples were collected.

\begin{tabular}{|llll|}
\hline & Case $(\mathrm{n}=15)$ & $\mathrm{C}(\mathrm{n}=15)$ & $\mathrm{P}$ \\
\hline Male & $4(26.7 \%)$ & $5(33.3 \%)$ & 0.7 \\
\hline Years & $73.5 \pm 12.1$ & $70.5 \pm 7.1$ & 0.6 \\
\hline Age schooling & $11.3 \pm 5.0$ & $11.3 \pm 2.2$ & 0.2 \\
\hline MMSE & $19.1 \pm 6.0$ & $28.1 \pm 1.8$ & 0 \\
\hline Family history & & & \\
\hline Presenilin AD & $2(13.3 \%)$ & $0(0 \%)$ & 0.14 \\
\hline EA >65 years & $3(20.0 \%)$ & $5(33.3 \%)$ & 0.4 \\
\hline Pathological history & & & \\
\hline Hypertension & $6(40 \%)$ & $8(53 \%)$ & 0.46 \\
\hline Diabetes & $3(20.0 \%)$ & $2(13.3 \%)$ & 0.6 \\
\hline Hypercholesterolemia & $2(13.3 \%)$ & $7(46.7 \%)$ & 0.04 \\
\hline Depression & $2(13.3 \%)$ & $7(46.7 \%)$ & 0.04 \\
\hline CSF AD Biomarkers & $\mathrm{pg} / \mathrm{ml}$ & $\mathrm{pg} / \mathrm{ml}$ & \\
\hline Amyloid $\beta$ & $385.6 \pm 128.1$ & $856.2 \pm 204.3$ & 0 \\
\hline Total Tau & $614.8 \pm 270.2$ & $280.7 \pm 103.9$ & 0.01 \\
\hline PhosphoTau & $84.5 \pm 27.7$ & $55.4 \pm 17.9$ & 0 \\
\hline
\end{tabular}




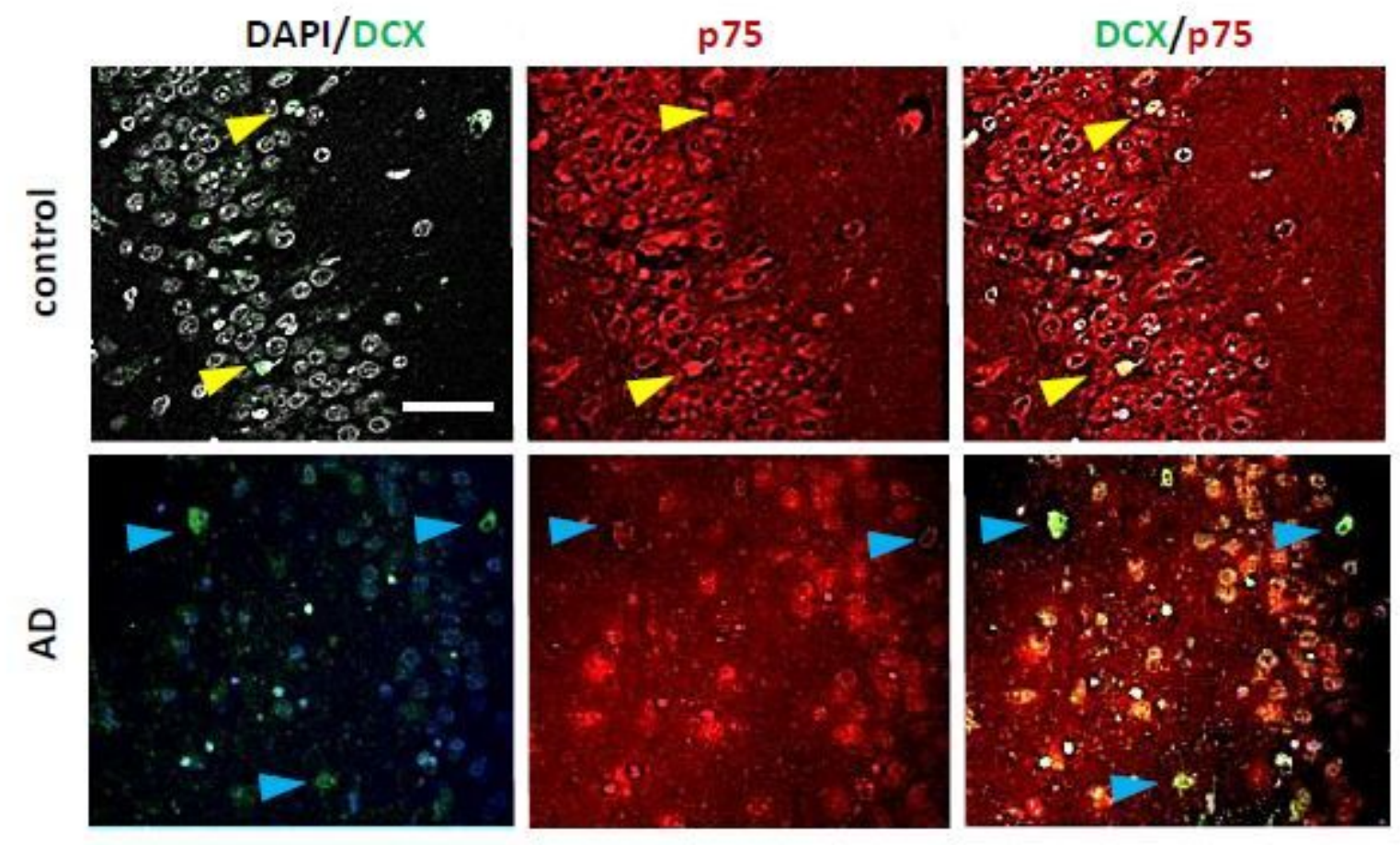

B

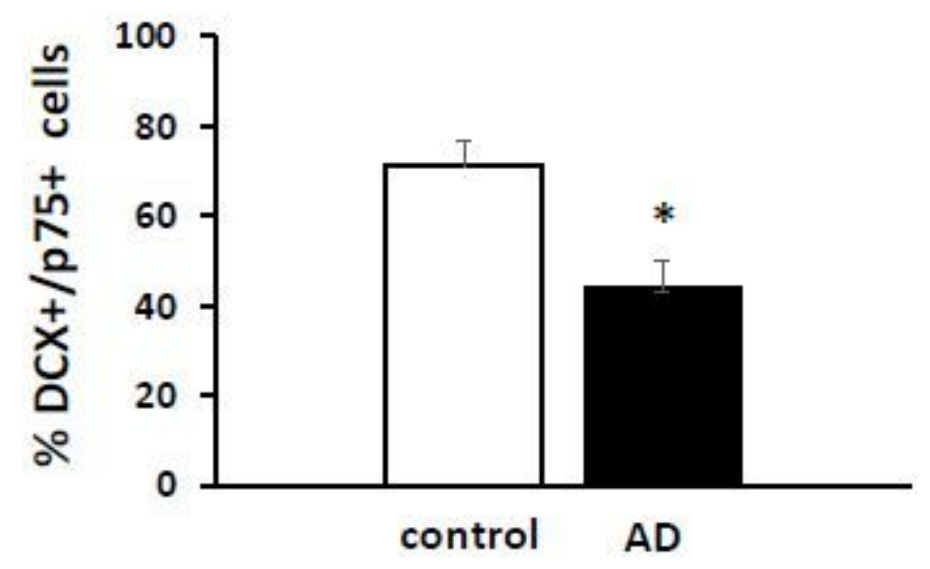

Figure 1

DCX and P75NTR expression in the human DG. (A) Representative confocal images of the neurogenic positive cells for DCX (green), p75NTR (red), and DAPI (grey) in DG of human samples of AD and control patients. Control (C) shows greater colocalization of DCX and p75 than AD samples (yellow arrows). Blue arrows indicate DCX+ and p75- cells. Scale bar $=50 \mu \mathrm{M}$. (B) Bars show the quantification of p75 and DCX colocalization. $A D$ shows a decreased colocalization of $D C X$ and $p 75$ with respect to controls. Bars are expressed as the mean of \% of p75NTR+ on the DCX + cells (DCX/p75NTR) in the DG of samples from 
controls and $A D$ affected patients. Positive cells are considered those with staining in the cell body. 300 total cells are counted from each sample. ( ${ }^{*}<0.05$; ANOVA), $(n=3$ control, $n=4$ AD).

A
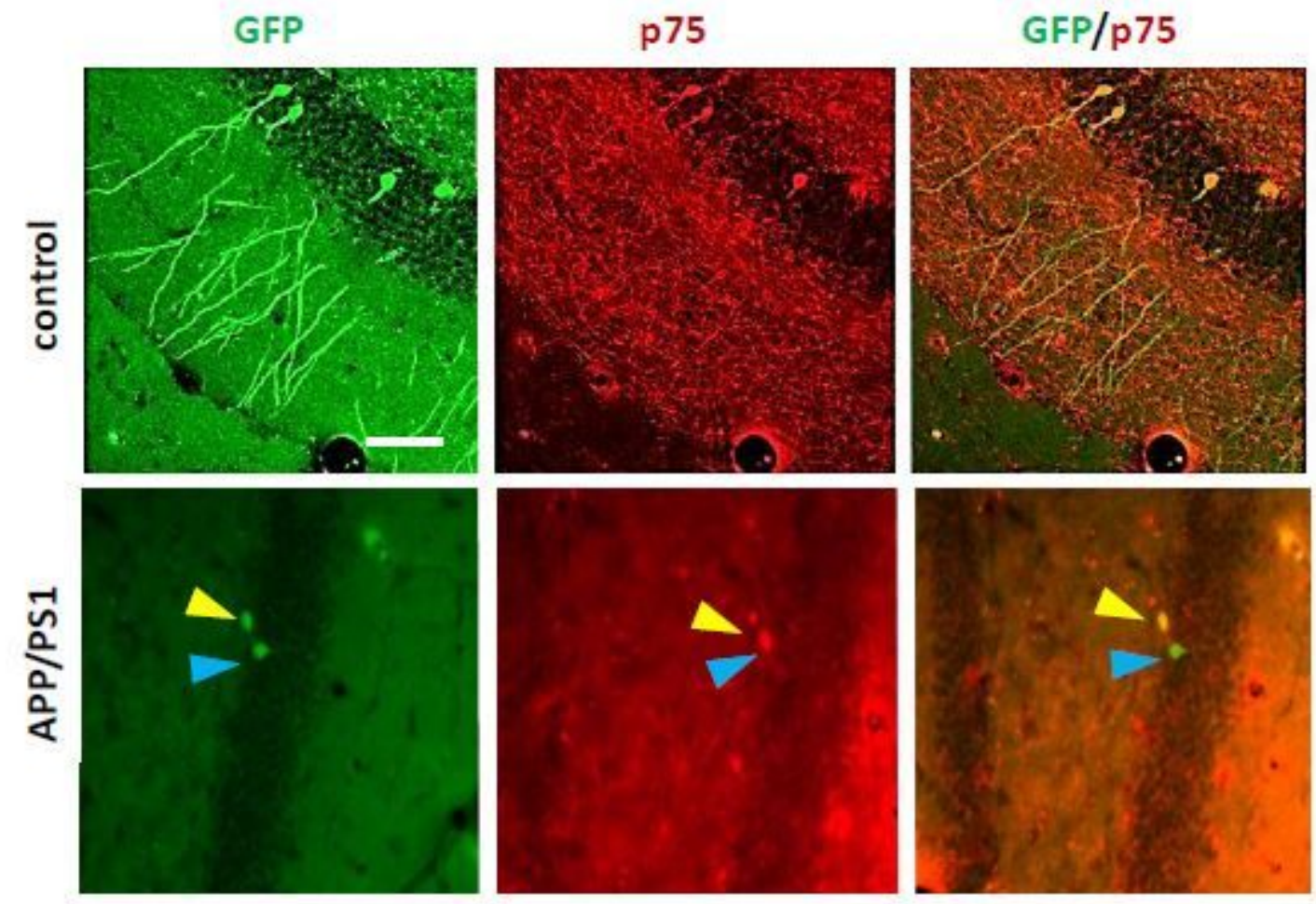

B

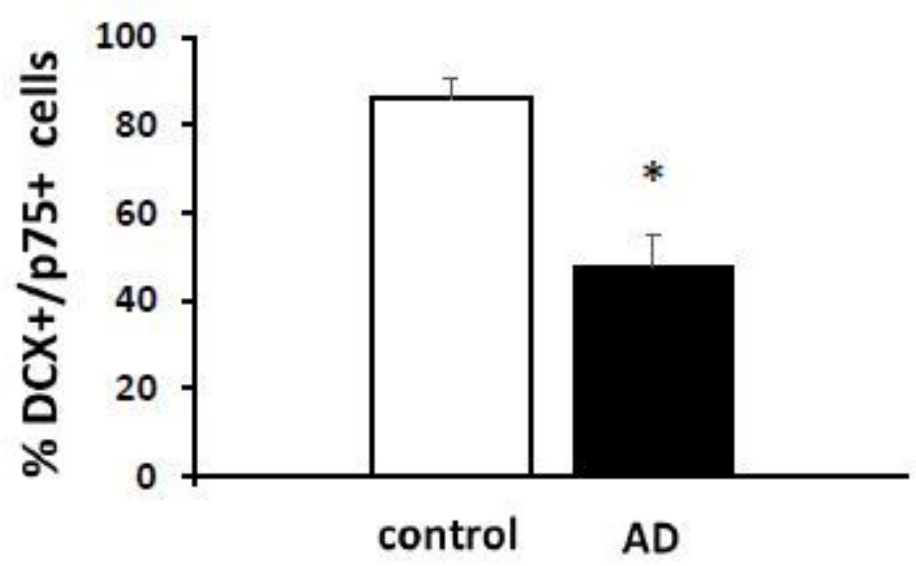

Figure 2

p75NTR expression in the DG of GFP retroviral injected mice. (A) Confocal images of the neurogenic cells transduced by GFP at 21dpi, GFP+ cells (green) expressing p75NTR (red), p75NTR and GFP colocalization (merge). Example of colocalization of DCX and p75 compared to AD samples (yellow arrows) and DCX+ and p75- cells (blue arrows). Scale bar $=25 \mu \mathrm{m}$. (B) Bars show decreased colocalization of GFP and p75 in APP/PS1 model as compared to control. Bars are expressed as the mean of \% of p75NTR+ on the DCX+ cells (DCX/p75NTR) in the DG of APP/PS1 mice and controls. Positive 
cells are considered those with staining in the cell body. 300 total cells from each sample. $(\mathrm{n}=5 \mathrm{mice}$ per group; ${ }^{* \star} p<0.01$; ANOVA).

A

\section{BSA}
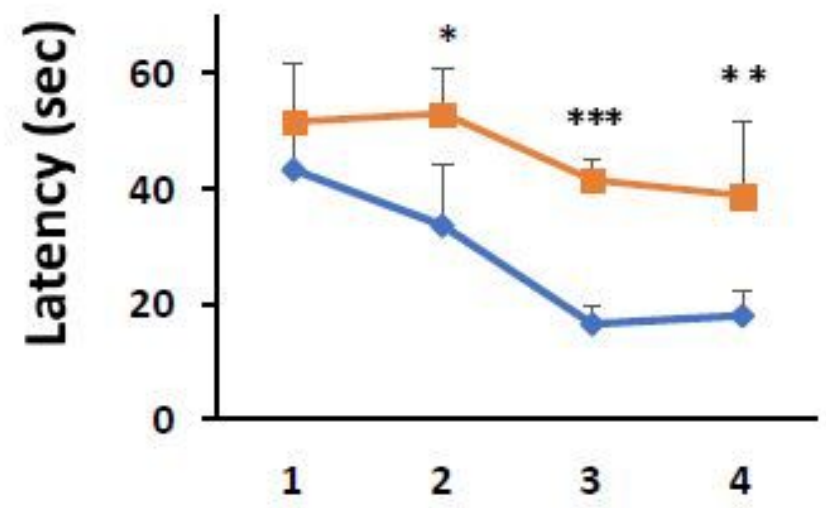

C

Control

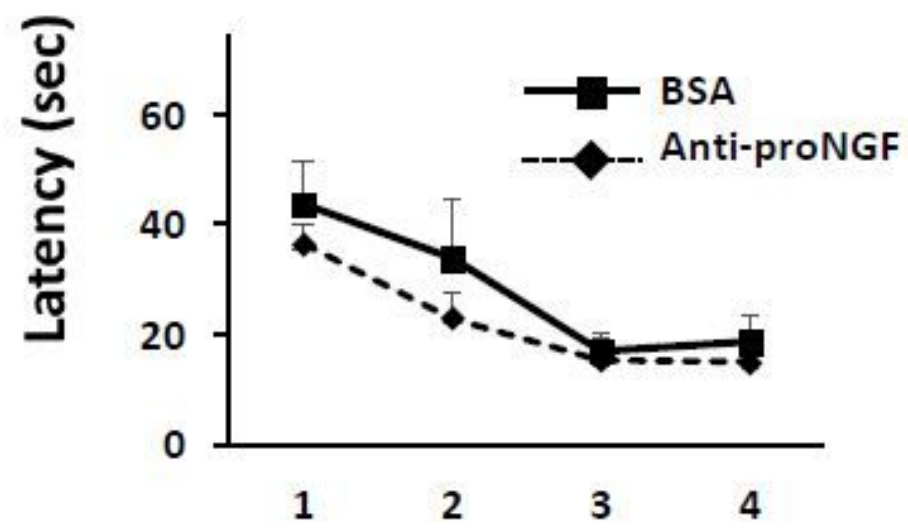

Trial day
B

anti-proNGF

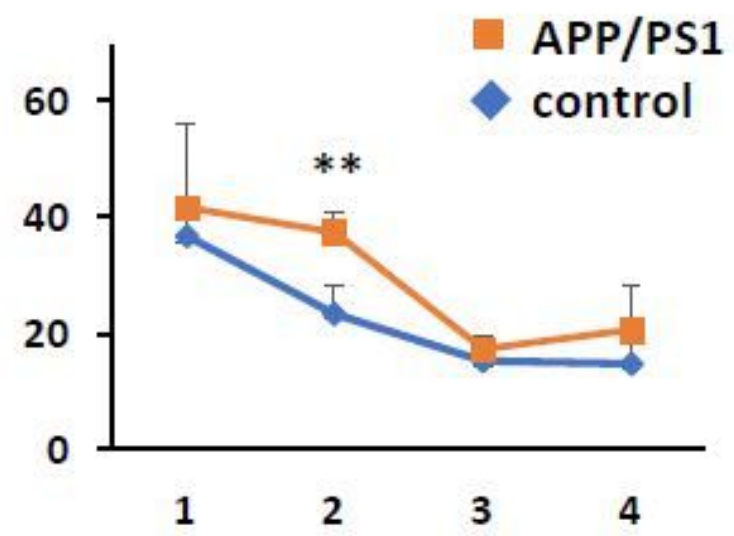

D

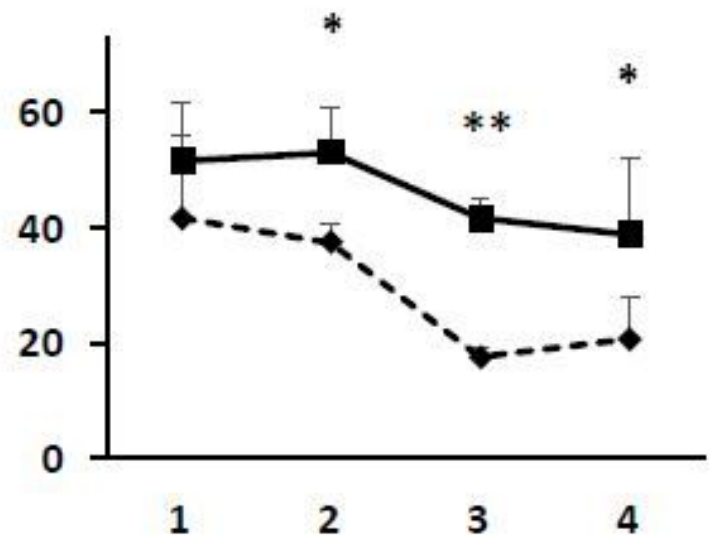

Trial day

Figure 3

The injection of anti proNGF antibody recovers the memory impairment in APP/PS1 mice (A) Morris water maze test in animals injected with BSA. Results show a higher latency in finding the hidden platform (latency (sec)) in the APP/PS1 transgenic mice (red line) compared to the control animals (blue line). (B) Morris water maze test in animals injected with anti-pro-NGF antibody. Results show a similar latency (sec) in the APP/PS1 transgenic mice (red line) compared to control animals (blue line). (C) 
Morris water maze test in control animals injected with BSA (solid line) or with anti-pro-NGF antibody (dashed line). Results show a similar latency in control animals injected either with BSA or with antiproNGF antibody. (D) Morris water maze test in APP/PS1 animals injected with BSA (solid line) or antipro-NGF antibody (dashed line). Results show a significantly higher latency (sec) in the APP/PS1 transgenic mice when injected with the antibody (dashed line) compared to the injected with BSA. ( $n=5$ mice per group; ${ }^{\star} p<0.05 ; * \star p<0.01, * \star \star p<0.001$ ANOVA). Figure 3 . The injection of anti proNGF antibody recovers the memory impairment in APP/PS1 Mice (A) Morris Water Maze Test in animals injected with BSA. Results show a higher latency (sec) in the APP/PS1 transgenic mice (red line) compared to the control (blue line). (B) Morris Water Maze Test in animals injected with anti pro-NGF antibody. Results show a similar latency (sec) in the APP/PS1 transgenic mice (red line) than control animals (blue line). (C) Novel Object Recognition Test in animals injected with BSA. Control animals have a higher index preference than APP/PS1 mice. (D) Novel Object Recognition Test in animals injected with anti proNGF antibody. Control and APP/PS1 animals do not differ in the index preference. $(n=5$ mice per group; * $p<0.05 ; * \star p<0.01, * \star * p<0.001$ ANOVA). 
A
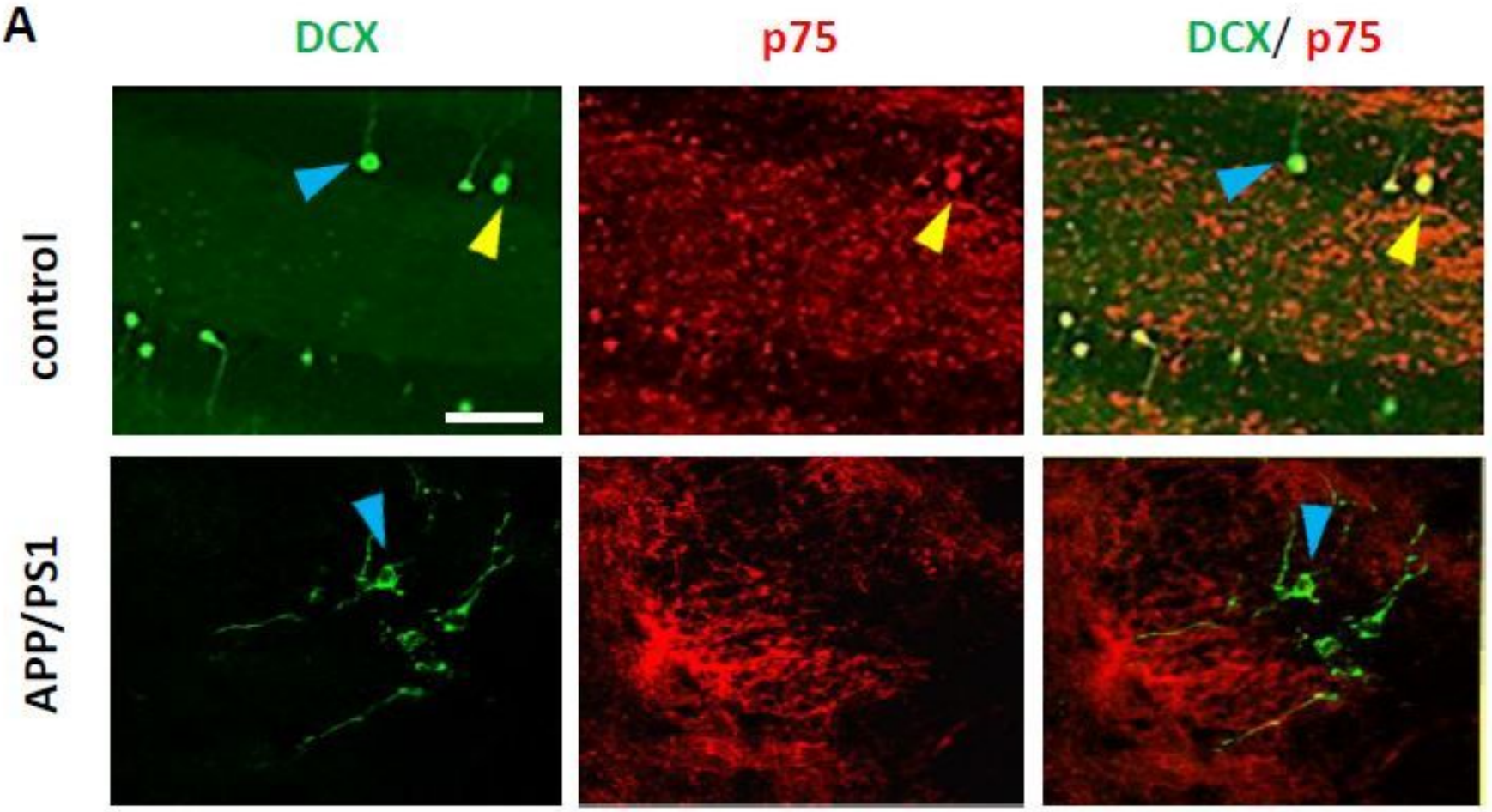

B

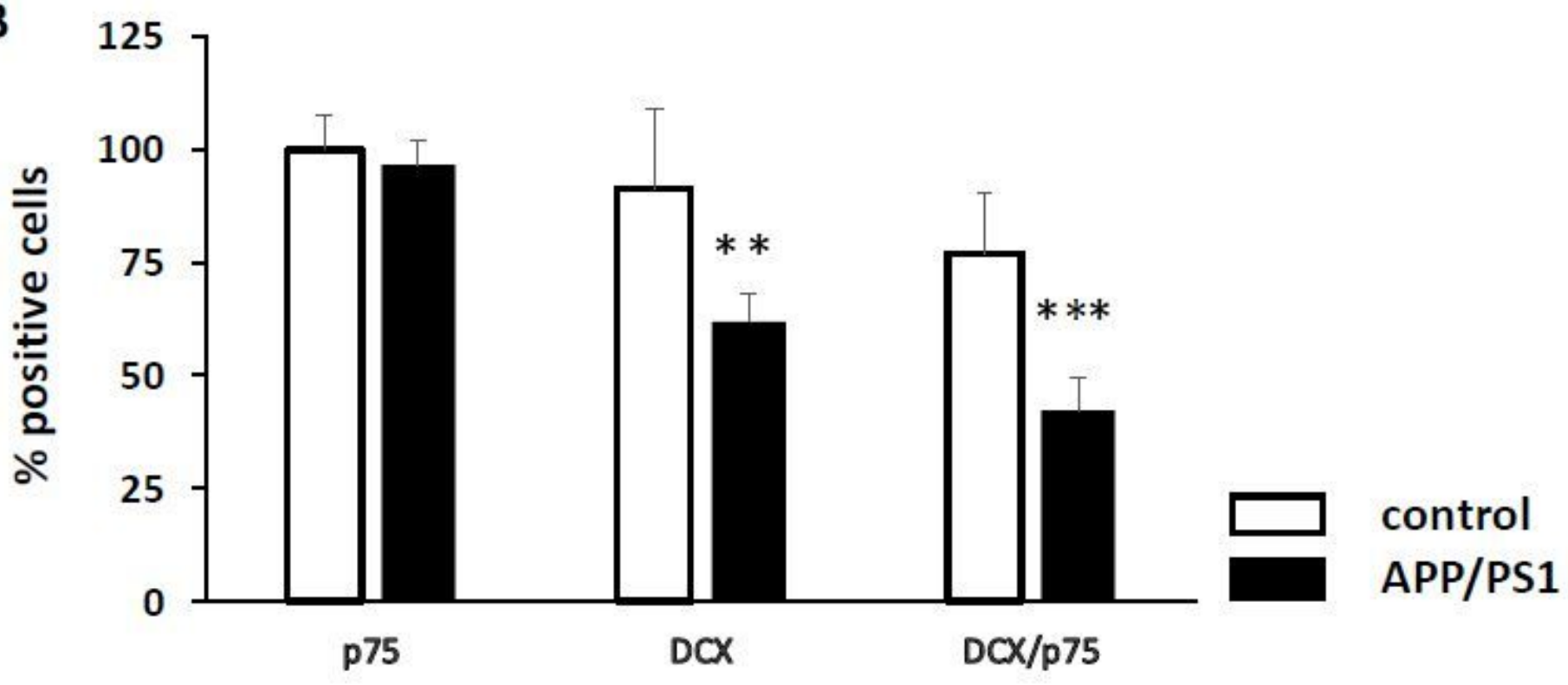

Figure 4

Neurogenesis in the DG of control and APP/PS1 mice in animals injected with control BSA. (A) Confocal images representing immunofluorescence pictures showing colocalization of $p 75$ and $D C X$ in the control and AD groups. p75 (red), DCX (green). Example of colocalization of DCX and p75 compared to AD samples (yellow arrows) and DCX+ and p75- cells (blue arrows). Control showing greater colocalization of DCX and p75 than the APP/PS1 transgenic mice. Scale bar $=25 \mu \mathrm{m}$. (B) Bars represent the mean of $\%$ positive cells \pm SD respect to total cells ( $p 75$ NTR and DCX) or \% of p75NTR+ on the DCX positive cells 
(DCX/p75NTR) in the DG of APP/PS1 mice and controls. Positive cells are considered those with staining in the cell body. 300 total cells from each sample. Values are expressed as the percentage of the highest mean ( $n=5$ mice per group; * $p<0.05$; ANOVA).

A
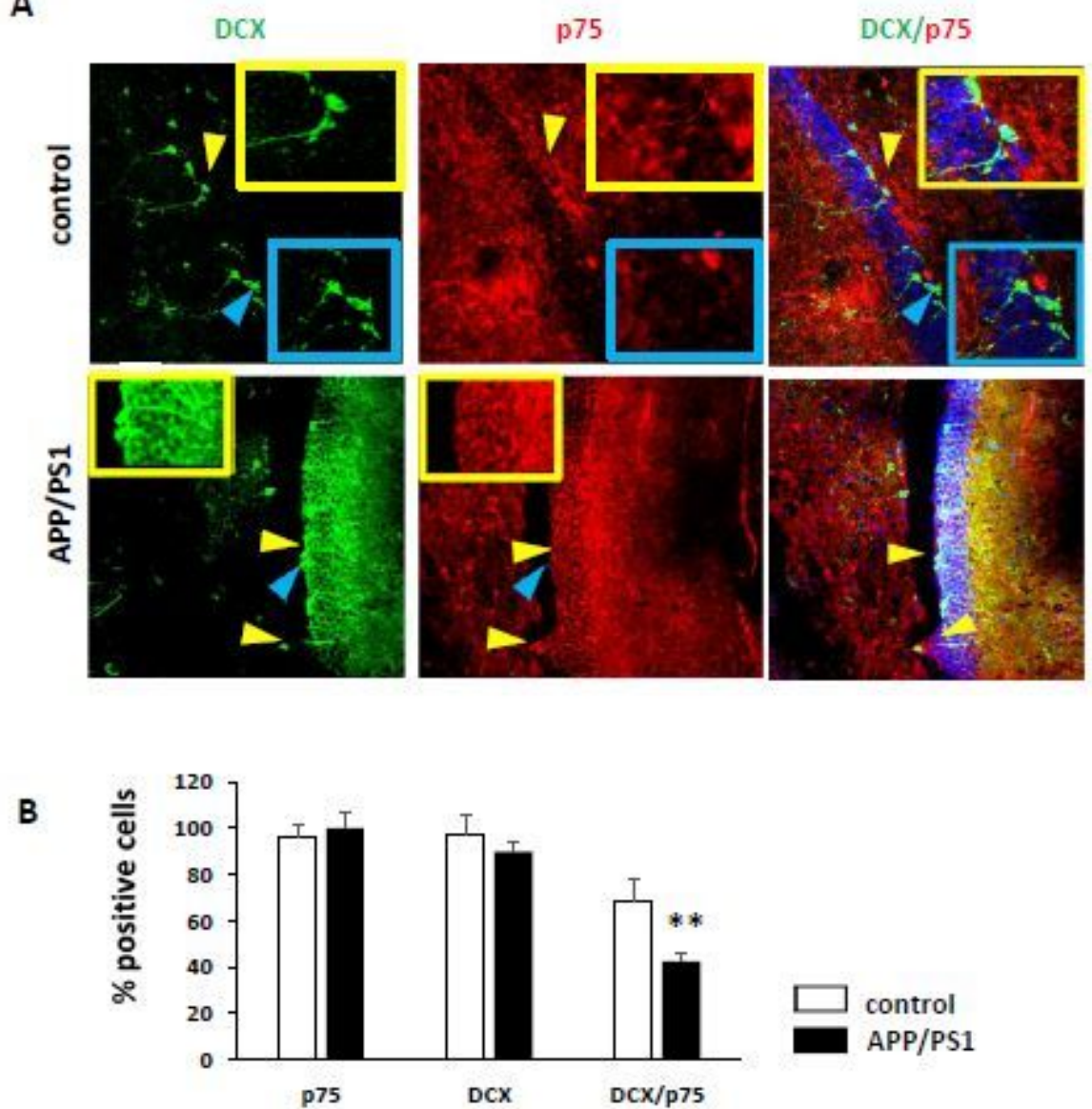

C

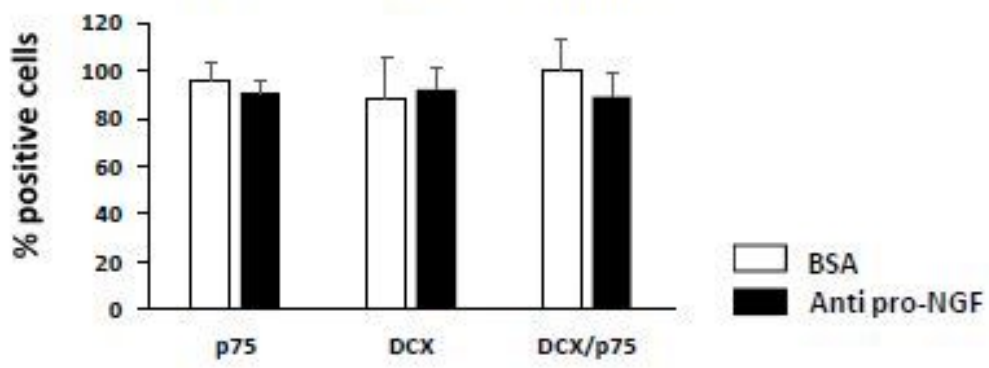

D

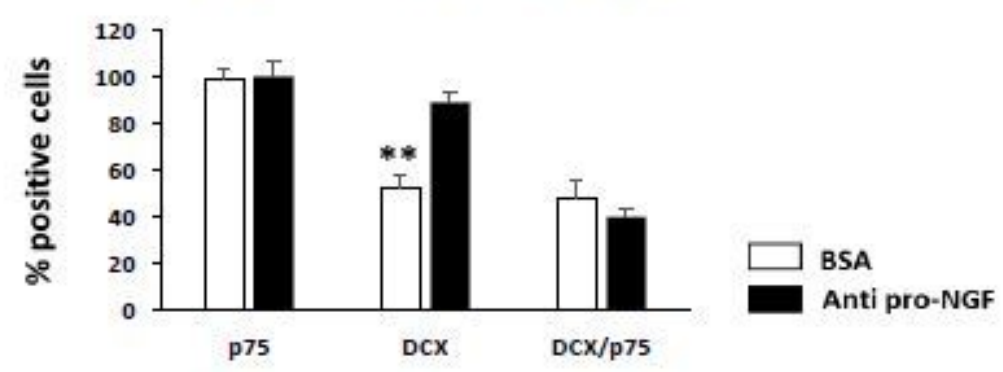

Figure 5

Neurogenesis is increased in the DG of APP/PS1m mice injected with anti-proNGF antibody. (A) Confocal images representing immunoflurorescence pictures showing colocalization of p75 and DCX in the control 
and AD groups. DAPI (blue), p75 (red), DCX (green). Example of colocalization of DCX and p75 compared to $A D$ samples (yellow arrows) and $D C X+$ and $p 75$ - cells (blue arrows). Controls showing greater colocalization of DCX and p75 than APP/PS1 transgenic mice. Scale bar $=50 \mu \mathrm{m}$. (B) Bars represent the mean of \% positive cells \pm SD respect to DAPI stained cells or $\%$ of $p 75 N T R+$ on the DCX positive cells (DCX/p75NTR) in the DG of APP/PS1 mice and controls. Positive cells are considered those with staining in the cell body. 300 total cells from each sample. (C) Comparison of BSA and anti-proNGF antibody injected into control mice. (D) Comparison of BSA and anti proNGF antibody injected into APP/PS1 mice. Values are expressed as the percentage of the highest mean. $\left(n=5\right.$ mice per group; ${ }^{*} p<0.05 ;{ }^{* \star} p<0.01$; ANOVA).

\section{Supplementary Files}

This is a list of supplementary files associated with this preprint. Click to download.

- SuppFig1.jpg 